Research Article

\title{
Simulation and Experiment of Dynamic Properties of Joint Surfaces Based on Fractal Theory
}

\author{
Haitao Liu, Yazhou Sun, and Zongshan Liu \\ Harbin Institute of Technology, School of Mechanical Engineering, P.O. Box 422, Harbin 150001, China \\ Correspondence should be addressed to Yazhou Sun; sunyzhit@163.com
}

Received 2 September 2014; Accepted 14 January 2015

Academic Editor: Nuno M. Maia

Copyright (c) 2015 Haitao Liu et al. This is an open access article distributed under the Creative Commons Attribution License, which permits unrestricted use, distribution, and reproduction in any medium, provided the original work is properly cited.

\begin{abstract}
Dynamic properties of joint surfaces are researched, micro behavior is also analyzed and a mathematical model based on fractal theory is built, and the relationships between normal dynamic characteristics of joints and surface pressure, surface roughness, and real contact area were simulated. The contact pressure in joint, equivalent stiffness, and damping in joint were nonstrict proportional relationship, higher surface quality of the contact joint surface, can increase normal stiffness and reduce normal damping in joint. Experiments are arranged according to the theoretical model in order to analyze the share of every major factor that affects dynamic properties of joint surfaces. Two common materials HT200 and 2Cr13 under different processing methods, surface roughness, and surface areas are used, and law curves were built between the dynamic behavior of fixed joints and preload, processing method of contact surface, surface roughness; the correctness of the theory simulation results was confirmed. A spring-damping element joints finite model was built based on the pressure distribution contours. Based on the experimental data, we simulated the model of HT200 specimen by ANSYS, at the same time, compared our model, traditional model, and experimental result, and proved that the spring-damping distribution model based on pressure has a better simulative precision.
\end{abstract}

\section{Introduction}

The joint surfaces are common in mechanical structures, and nonlinear characteristics of joint surfaces are the main factor which affects the dynamic behavior of whole machine. The method of mechanical design changes from static to dynamic with the trend that modern machinery has high speed, high precision, and light weight. Moreover, calculation of dynamic behavior of machines is the key point to dynamic design. Therefore, the research of dynamic characteristics on the joints is extremely vitally significant to the structure design and optimization [1-3].

The research of joint surfaces originates from the measurement of static stiffness. Scholars analyzed the laws of normal and tangential properties of joint surfaces according to experiments. After long-time accumulation, they built a database of static properties for joint surfaces [4]. On the basis of static properties, scholars have researched the stiffness and damp properties of joint surfaces according to theoretical model and experiment results and studied dynamic properties and parameter recognition methods of joint surfaces.
These works are of great importance to building a precise dynamic model.

Early research of the joint surfaces focused on the static properties of a few of common joint surfaces, and the error was huge. After that, many scholars carried out further research on dynamic properties of joint surfaces along with the factors that influence these dynamics. Both Oden and Martins [5], Schofield et al. $[6,7]$ and Sherif $[8,9]$ carried out experiments between under different pressure joint surfaces of real machine tools, results indicated that normal load and deload curves of joint surfaces under high pressure different from that of low pressure. They thought the normal stiffness of joint surfaces has nothing to do with plastic deformation but elastic deformation, the normal pressure is in linear proportion to elastic deformation. Both Yumei's [4] and Oden and Martins's [5] researches indicated that the factors affecting static properties of joint surfaces majorly incorporate material, processing methods of joint surfaces, surface roughness, real contact area, contact shape, pressure, and so forth. Contact faces bear normal and tangential forces simultaneously; Kirsanova [10] and Masuko et al. [11] furtherly studied 
tangential properties of joint surfaces and indicated that tangential stiffness is in nonlinear proportion to contact pressure; micro slip is responsible for tangential damp.

From 1970's, Europe, America, Japan, and so forth began to research the joint surfaces dynamic properties. Because the joint surfaces damp was not calculated and the value of system's damp was comparatively small, high-precision apparatus was needed to test the damping coefficient. Rogers and Boothroyd's [12] research indicated that joint dynamic contains both nonlinear and hysteresis properties, and the hysteresis property is caused by damp. Smallwood et al. [13] worked out a plan to measure the energy consumption of joint surfaces, proving that micro skip is the major cause of tangential damp.

Bograd et al. [14] summarized three different methods of building the dynamic model of joint surfaces: one based on Jenkins friction theory; one based on thin layer element simulation of dynamic properties; and the other one based on zero-thickness finite element simulation of dynamics. The author compared the advantages and disadvantaged of these methods in practical applications and proposed the idea of using different method when facing different loading conditions.

\section{Theoretical Modeling of Joint Surfaces}

Micro analysis majorly studies the stiffness and damp of joint surfaces by analyzing the micro surface and their deformation mechanism; the deformation mechanism of a single asperity is studied firstly, and the variation pattern of stiffness and damp of joint surfaces is analyzed according to fractal theory. This paper utilizes fractal theory to describe the outline of contact area, analyzes one single asperity's deformation at first, and then expands to the whole contact area. This method can reflect the microscopic contact state of contact area and provide theoretical basis for parameter recognition of joint surfaces.

2.1. Normal Stiffness Model of Joint Surfaces. Assuming that a coarse surface contacts with an ideal rigid plane, coarse surface with a surface roughness, has a corrugated structure, shown in Figure 1. Because the size and distribution of asperities are random, contact point size and distribution function $n(a)$ and the real contact area were introduced $A_{r}$ [15]:

$$
\begin{aligned}
& A_{r}=\int_{a_{s}}^{a_{m}} n(a) a d a \\
& n(a)=\frac{D}{2} \frac{a_{m}^{D / 2}}{a^{(D / 2)+1}}
\end{aligned}
$$

where $a_{m}$ is maximum area of contact area $\left(\mathrm{mm}^{2}\right)$; $a_{s}$ is minimum area of contact area $\left(\mathrm{mm}^{2}\right) ; D$ is fractal degree, $1<D<2$.

Focusing on one single asperity $X$, we assume its crosssection is circular, shown in Figure 2 . Assuming contact area of asperity $X$ is $a$ and $a \approx l^{2}$, the principle of fractal theory is to determine section area $a$ of asperity and calculate

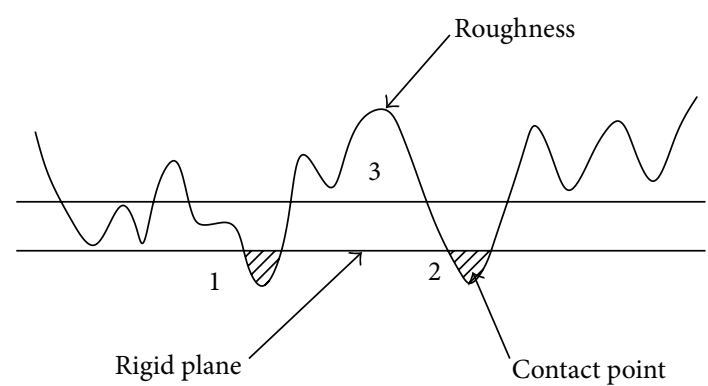

FIGURE 1: Schematic diagrams.

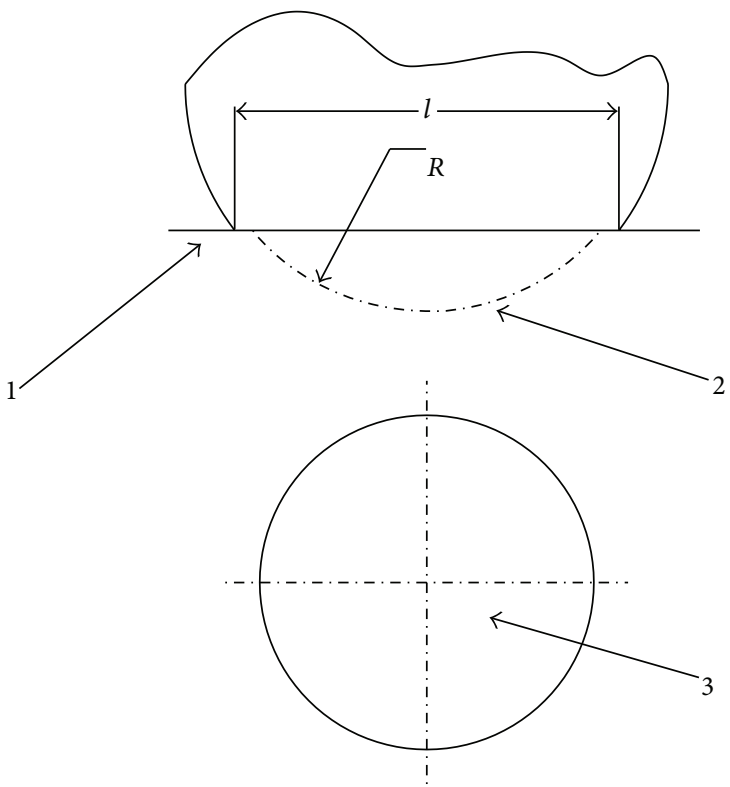

(1) Ideal rigid plane

(2) Asperity before deformation

(3) Cross-section after deformation

FIGURE 2: Schematic diagram of the contact of a single asperity.

the required deformation $\delta$, and then the required force $P$ is calculated based on Hertz theory.

According to $W-M$ model, the topography function $z(x)$ before deformation is [16]

$$
z(x)=G^{D-1} l^{2-D} \cos \left(\frac{\pi x}{l}\right) \quad-\frac{l}{2}<x<\frac{l}{2},
$$

where $G$ is fractal coarse coefficient, $D$ is the degree of fractal, and $1<D<2$.

It is obvious that $z$ reaches its peak $\delta$ when $x=0$ :

$$
\delta=z(0)=G^{D-1} l^{2-D}=G^{D-1} a^{(2-D) / 2} .
$$

The radius of asperity curvature before deformation is

$$
R=\left|\frac{1}{\left|\partial^{2} z / \partial x^{2}\right|_{x=0}}\right|=\frac{l^{D}}{\pi^{2} G^{D-1}}=\frac{a^{D / 2}}{\pi^{2} G^{D-1}} .
$$




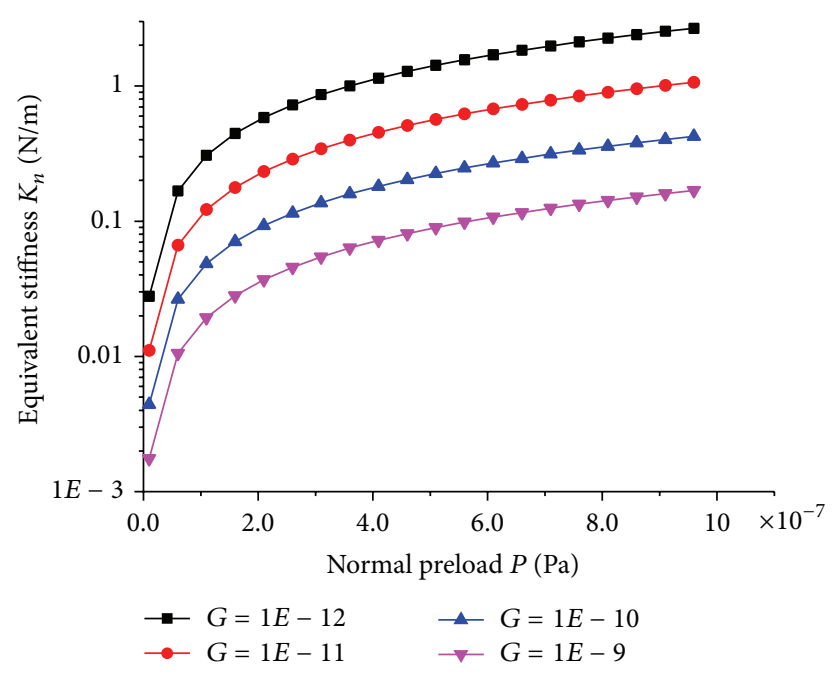

FIgURE 3: Equivalent normal stiffness of $2 \mathrm{Cr} 13$ joint surfaces.

According to Hertz theory, elastic deformation happened to asperity; the load $P_{e}$ is

$$
P_{e}(\delta)=\frac{4}{3} E_{n} R^{1 / 2} \delta^{3 / 2} .
$$

When deformation $\delta$ happens to an asperity, according to (6), the normal stiffness is obtained:

$$
k_{n}=\frac{d P_{e}}{d \delta}=2 E_{n} R^{1 / 2} \delta^{1 / 2}=\frac{2 E_{n}}{\pi} a^{1 / 2} .
$$

By substituting (2) into (7), the normal stiffness $K_{n}$ can be obtained:

$$
K_{n}=\int_{a_{c}}^{a_{m}} k_{n} n(a) d a=\frac{2 D E_{n}}{(1-D) \pi}\left(a_{m}^{1 / 2}-a_{m}^{D / 2} a_{c}^{(1-D) / 2}\right),
$$

where $E_{n}$ is composite modulus of elasticity; $1 / E_{n}=(1-$ $\left.v_{1}^{2}\right) / E_{1}+\left(1-v_{2}^{2}\right) / E_{2}, E_{1}, E_{2}$ are modulus of elasticity of the two materials which form the joint surfaces. $v_{1}, v_{2}$ are Poisson ratio.

According to (8), $K_{n}$ depends on material $\left(E_{n}\right)$, topography $(D)$ and roughness $\left(a_{m}\right)$, and so forth. The simulation result of normal stiffness is based on (8) and (6) is shown in Figure 3. $K_{n}$ increases with the increasing of $P$. When $P$ is low, stiffness grows faster and then gradually tends to slow, and $K_{n}$ decreases with the increasing of $G$. By combining (1), (2), and (6), the relationship between normal stiffness $K_{n}$ and real contact area $A_{r}$ can be obtained, shown in Figure 4. The normal stiffness of joint surfaces increases along with real contact area, but the gradient of the curve decreases which means the increasing rate would drop while real contact area increases.

The relationships of normal stiffness between fractal degree and material are shown in Figure 5. Figures 5(a) and 5(b) indicate that normal stiffness increases along with the surface quality and the proportion of high frequency profile. Meanwhile Figure 5(c) implies the feeble influence of material type on the normal stiffness of joint surfaces.

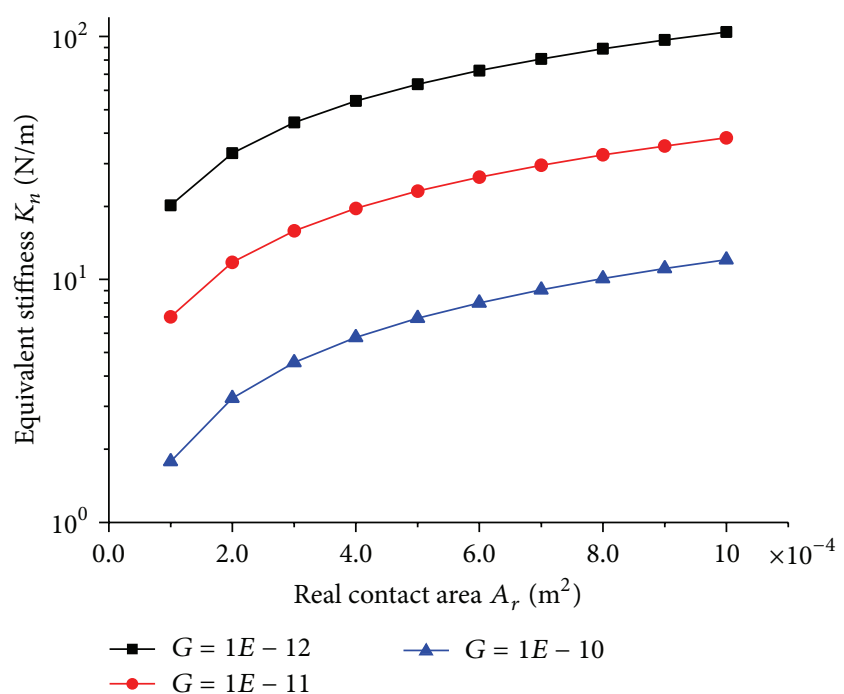

Figure 4: Relationship between $K_{n}$ and $A_{r}$ when $D=1.4$.

2.2. Fractal Model of Normal Damping. We assume that the plastic deformation of asperities dominates the normal damping. When calculating the damping coefficient of joint surfaces, contact area can be simply divided into elastic and plastic regions. By analysis of the strain energy caused by elastic deformation, energy consumption of plastic region $\eta$ can be obtained:

$$
\eta=\frac{15 H(5-3 D) a_{C}^{2-D}}{8 E(2-D) G^{D-1}\left(a_{m}^{(5-3 D) / 2}-a_{C}^{(5-3 D) / 2}\right)},
$$

where $H$ is the hardness of soft material.

According to Hertz theory, in plastic region, the deformation of a single asperity is in proportion to load and its section area:

$$
P_{p}=H a .
$$




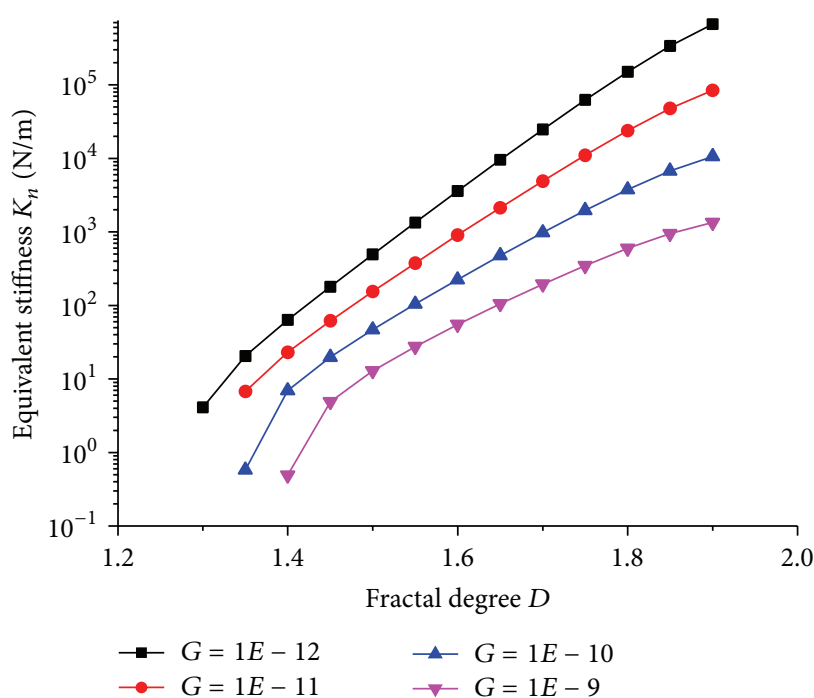

(a) $2 \mathrm{Cr} 13$

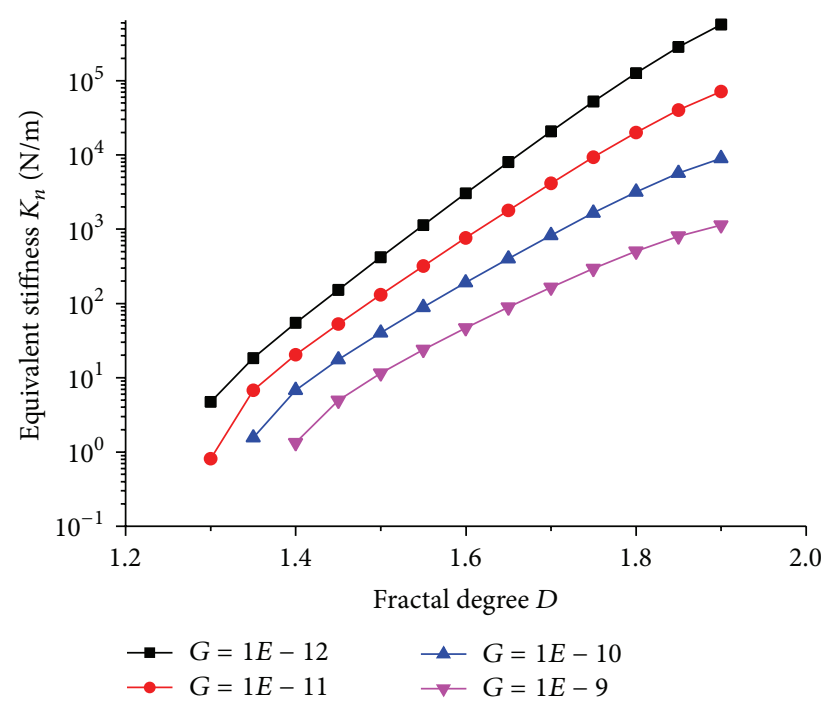

(b) HT200

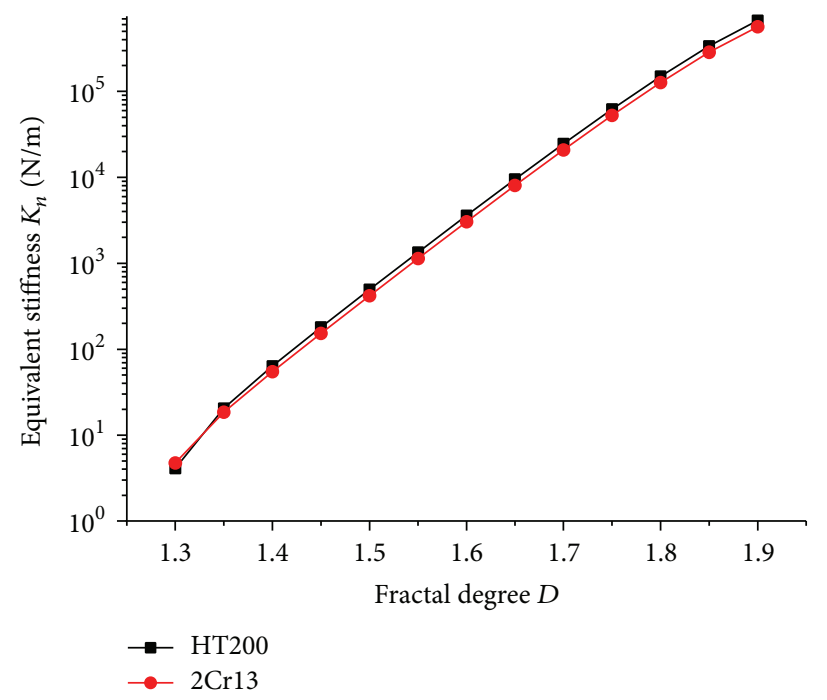

(c) Comparison of different material

FIGURE 5: Relationship of equivalent stiffness with fractal degree $D$.

And the total load of plastic region is

$$
P_{2}=\int_{0}^{a_{c}} \operatorname{Han}(a) d a=\frac{H D a_{m}^{D / 2}}{D+2} \cdot a_{c}^{(D+2) / 2} .
$$

Simulation of $\eta$ with MATLAB shows the relationship between $\eta$ and $D$ in Figure 6. By substituting (9) into (10), the relationship between $\eta$ and $P$ is obtained, shown in Figure 7. Obviously, $\eta$ is hardly affected by normal stress.

To sum up, by analyzing microscopic fractal model of joint surfaces, the following conclusions can be obtained.

Equivalent normal stiffness and damping coefficient of joint surfaces depend on material, normal stress, surface roughness processing methods, and actual contact area. The relationship is nonlinear, especially for that of normal damping coefficient.
The most significant factors that affect joint surfaces' dynamics are normal stress, surface roughness, and topography. Normal stiffness increases along with normal stress; normal damping coefficient decreases slightly when normal stress increases; the better the surface quality is, the higher the normal stiffness is; the more uniform the topography is, the larger the $D$ is; when the normal stiffness becomes higher, meanwhile the normal damping coefficient increases to a certain value and then decreases.

\section{Experimental Analyses}

3.1. Experiment Set-Up. The experiment set-up shown in Figure 8 is specially designed to analyze the effect of external factors (including external load and participating media) and major intrinsic factors (including material, surface roughness, processing method, and dimension of joint faces) 


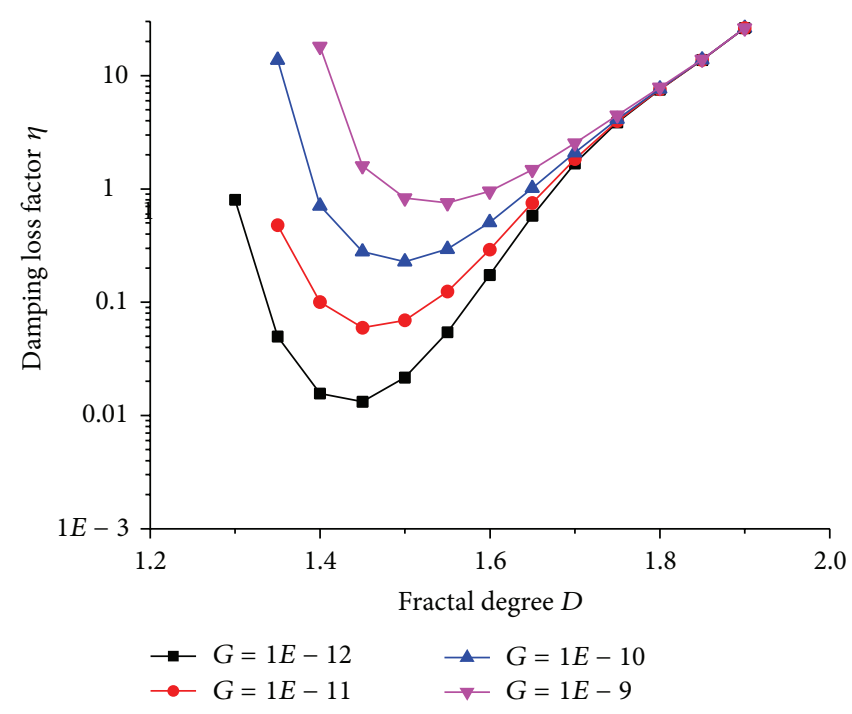

Figure 6: Relationships between $\eta$ and $D$.

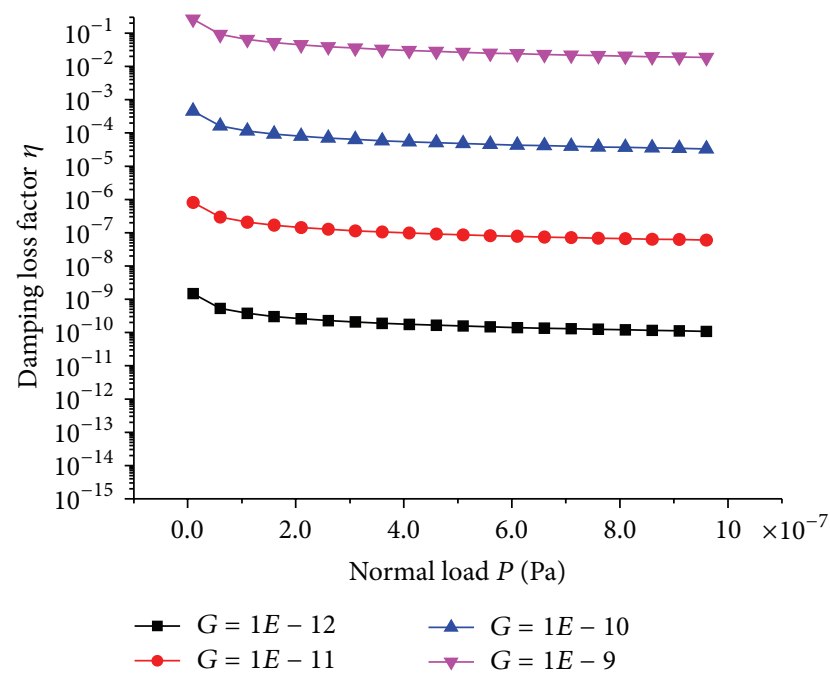

FIgURE 7: Relationships between $\eta$ and $P$.

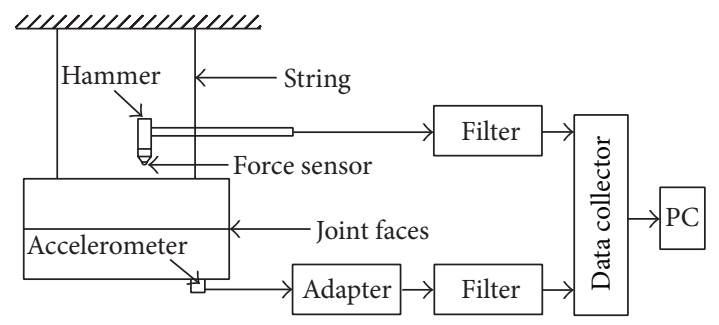

FIGURE 8: Experiment set-up.

according to the dynamic behavior of joint surfaces. Dynamic factors are also recognized according to series of experiments. Work pieces are suspended by string so that they will not be affected by external mass. The work pieces thus get 6 rigid vibration modes (including three translational and three rotational). Since the work pieces are small, a hammer is

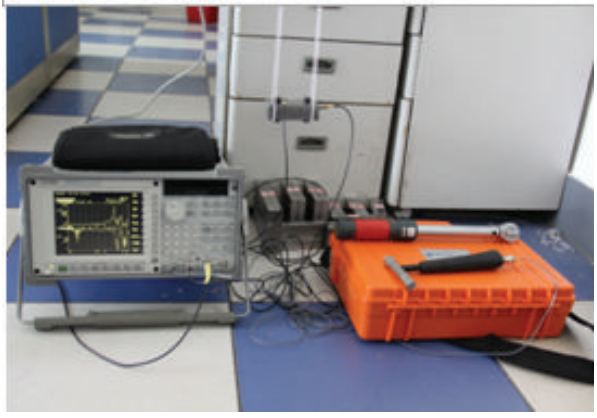

FIGURE 9: Experiment equipment.

chosen to excite the vibration; the vibration signal is detected by accelerometer and collected after noises are filtered.

Equipments used in this paper are shown in Figure 9.

3.2. Experiment Results. Figure 10 implies the cripple effect of joint faces on normal stiffness and enhancement of damping; it also implies that the dynamic properties of joint faces can be improved by increasing preload or surface quality.

Figure 11 reveals the relationship between surface roughness, normal stiffness, and damping. It indicates that the dynamic properties of joint faces increase while surface roughness decreases.

Figure 12 compares the normal stiffness and normal damping of joint faces under different contact areas. The one with smaller contact area has higher equivalent normal stiffness and overall lower damping coefficient.

\section{Improving Simulation Precision}

FEM plays a more and more important role in modern machine design. There are many methods in dealing with joint surfaces in a FEM model including glue, contact element, combining elements, and so forth. The first one simply 


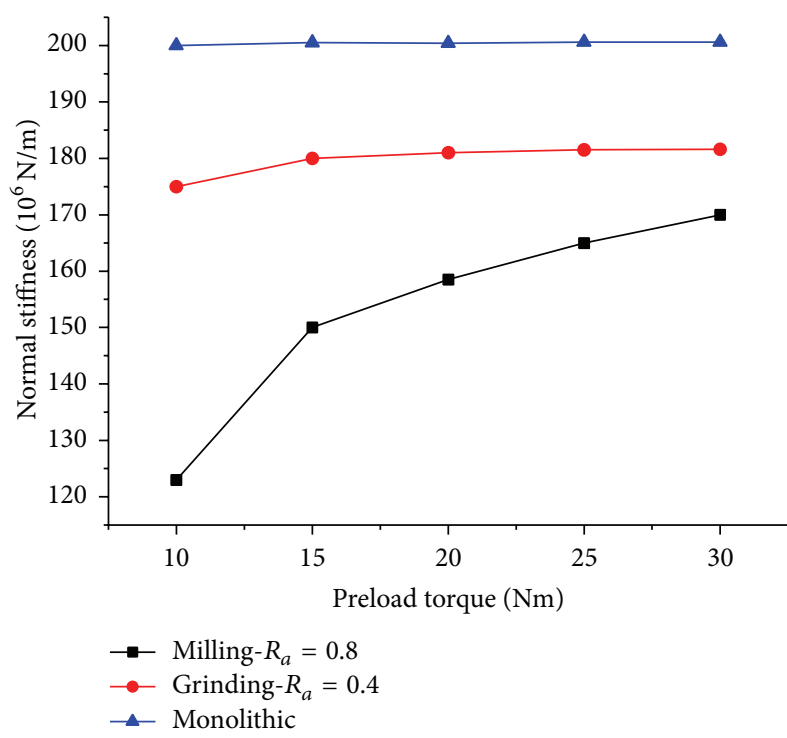

(a) Preload-normal stiffness

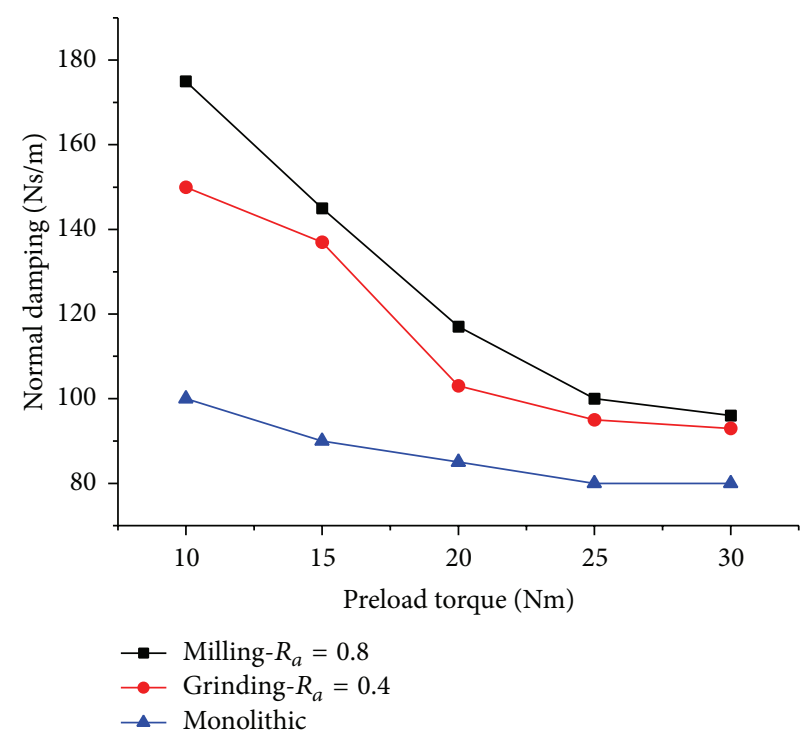

(b) Preload-normal damping

FIGURE 10: Analysis of normal dynamic characteristics.

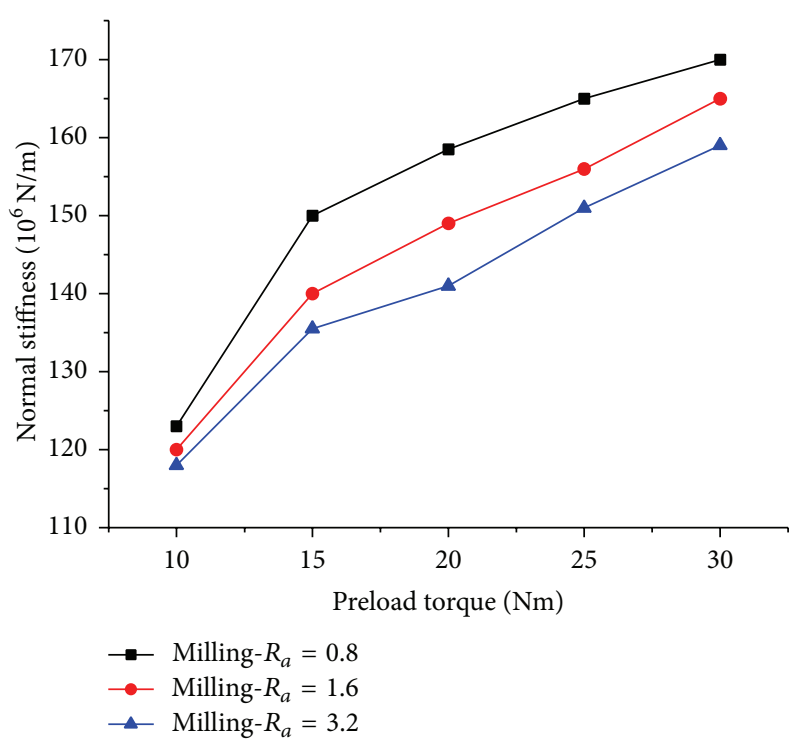

(a) Preload-normal stiffness

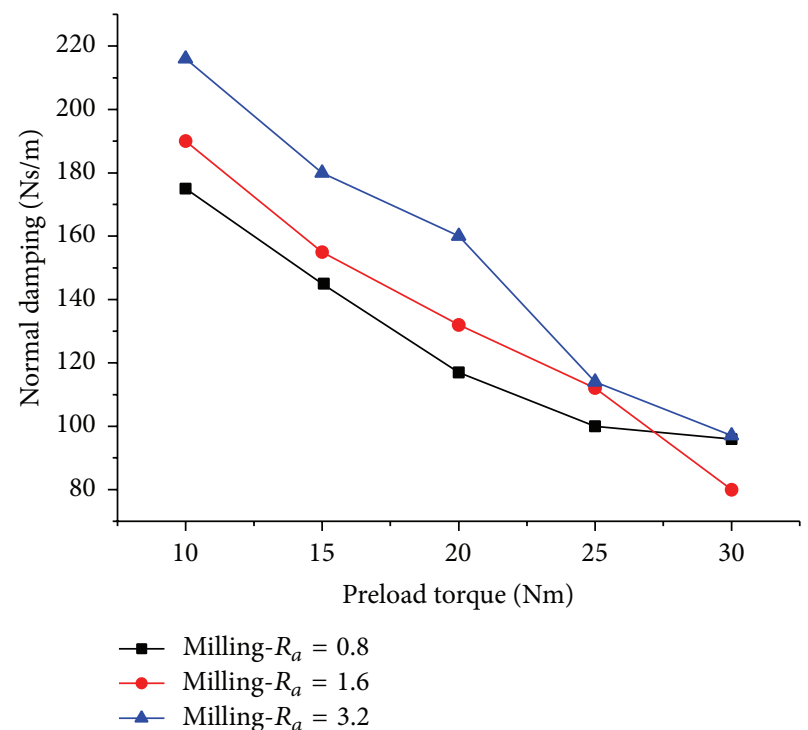

(b) Preload-normal damping

FIGURE 11: Comparison of dynamic characteristics in joint of different roughness.

combines two near nodes of two contact bodies together and makes the two bodies a monolithic one; this method, in fact, neglects the joint surface completely. Contact element method, however, assumes that joint surfaces keep uniform contact all the time and the stress is equal everywhere. This method is highly nonlinear and demands huge compute resources. What is more, it cannot ensure the convergence due to complicated calculation. The combined element method is hitherto believed to be the most effective way to simulate the joint surfaces, but the stiffness and damping coefficient of combined elements and their distribution may affect the simulation precision of joint surfaces significantly. This paper comes up with a pressure-dependent simulation method to improve simulation precision which has been proved to be more effective than traditional ways.

Figure 13 shows the FEM model of work pieces jointed with bolts (bolts are not shown in this figure) and stress distribution of joint surfaces. It can be proved by FEM simulations that when normal stiffness of combined elements in the FEM model is around the real stiffness of joint surfaces, the stress distribution will not be affected too much by stiffness variation of combined elements. According to theoretical analysis and experimental results, stiffness of joint surfaces is comparatively sensitive to normal stress. In this section, joint surface is divided into 3 regions, a, b, and c, shown in Figure 13(b). Element combin 14 is used and different stiffness 


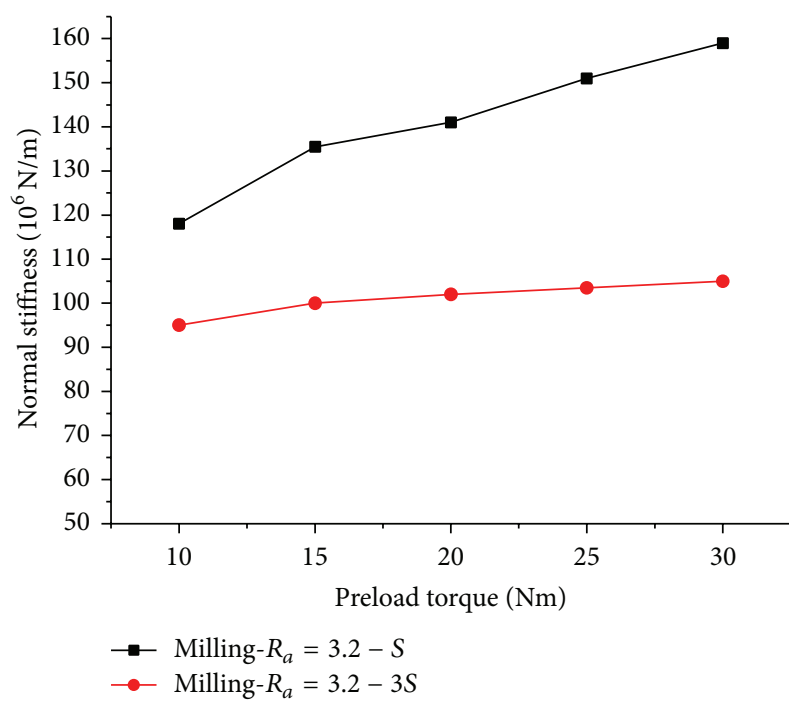

(a) Preload-normal stiffness

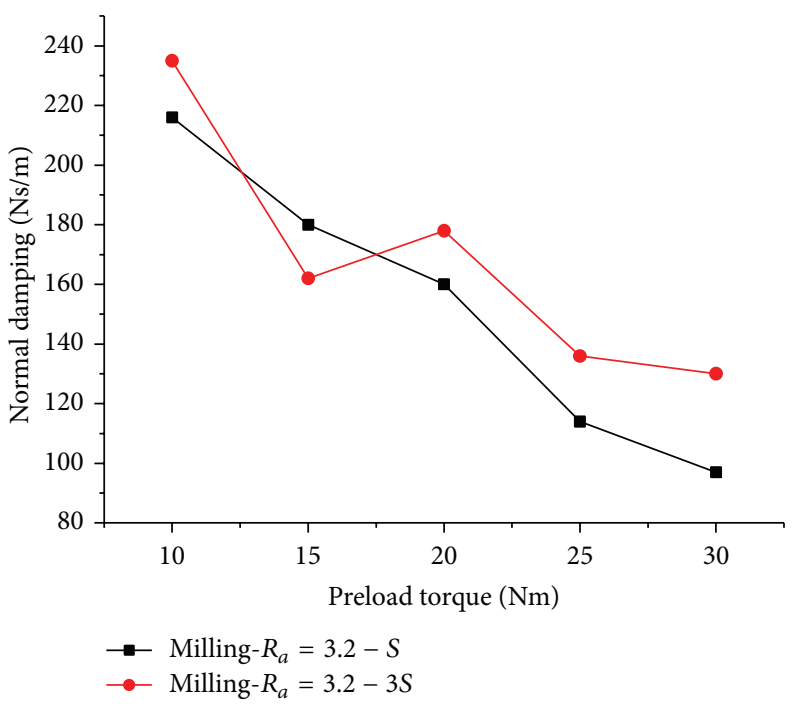

(b) Preload-normal damping

FIGURE 12: Comparison of dynamic characteristics in joint of different contact area.
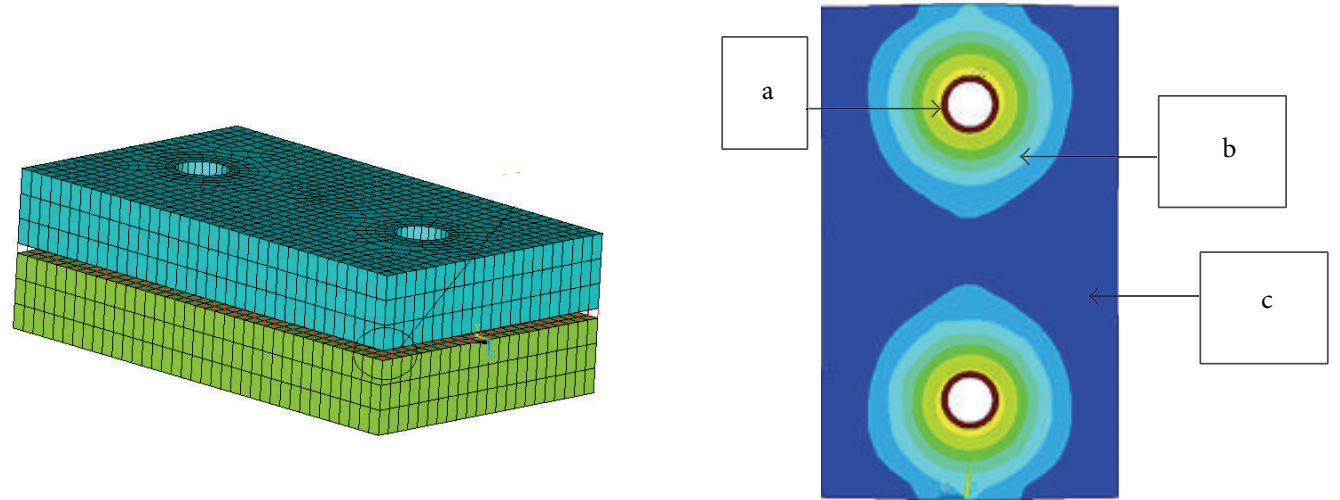

(a) Finite element model

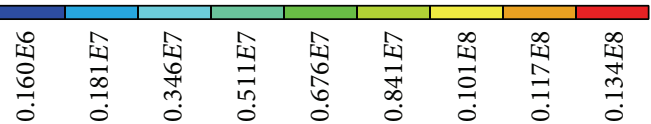

(b) Stress cloud

FIGURE 13: FEM model and stress distribution.

and damping constants are applied to elements in different regions.

In order to analyze the influence of stress distribution on simulation precision, another simulation model called even model is built in which all the stiffness and damping constants are equal. Limited by space, modal analysis results of stressdependent model are listed in Figure 14; the contrast model has very similar modal properties with stress-dependent models.

Figure 15 shows the simulation results of two different models: (a) shows the results under preload $10 \mathrm{Nm}$ and (b) shows results under preload $15 \mathrm{Nm}$. Obviously, stressdependent model shows better performance than the other one, especially when normal preload increases.

\section{Conclusions}

Normal dynamic property of joint faces is discussed here. By theoretical analysis, the conclusions depicted below are reached and verified by experiments.

(1) Joint mathematical model based on fractal theory can well describe the relationship between normal and tangential dynamic characteristics of joints and surface pressure, surface roughness, and real contact area.

(2) Equivalent normal stiffness and damping of joint surfaces depend on material, normal stress, surface roughness, processing methods, and actual contact 


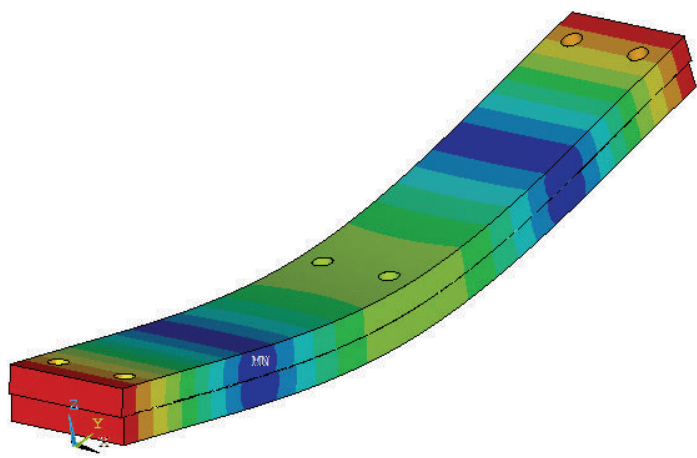

$\begin{array}{llllllllll}0.002 & 0.085 & 0.169 & 0.352 & 0.335 & 0.419 & 0.502 & 0.589 & 0.669 & 0.753\end{array}$

(a) 1st vibration mode

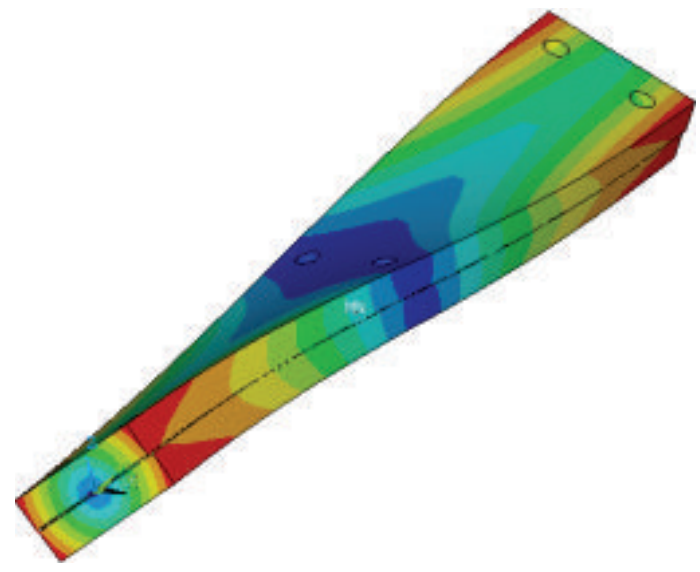

$\begin{array}{lllllllllll}0.001 & 0.184 & 0.286 & 0.312 & 0.415 & 0.519 & 0.613 & 0.717 & 0.831 & 0.934\end{array}$

(c) 3rd vibration mode

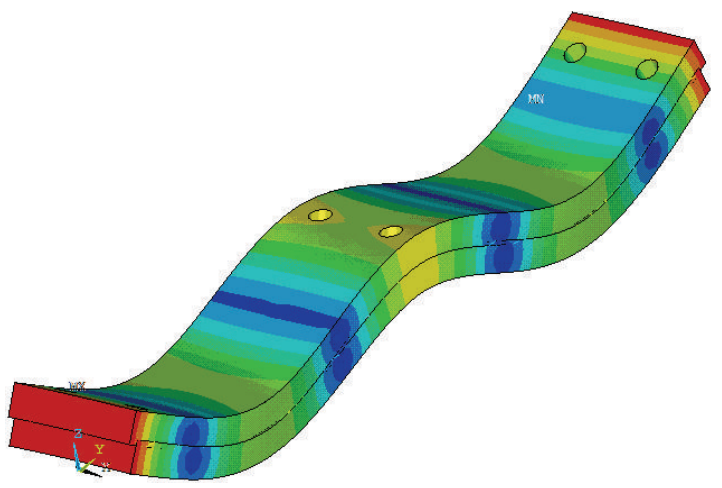

$\begin{array}{llllllllll}0.019 & 0.103 & 0.186 & 0.270 & 0.354 & 0.438 & 0.521 & 0.603 & 0.689 & 0.772\end{array}$

(e) 5 th vibration mode

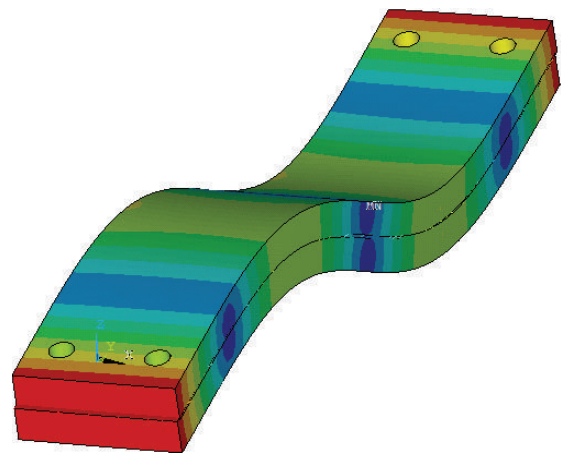

$\begin{array}{llllllllll}0.192 & 0.098 & 0.177 & 0.355 & 0.334 & 0.413 & 0.492 & 0.571 & 0.650 & 0.729\end{array}$

(b) 2nd vibration mode

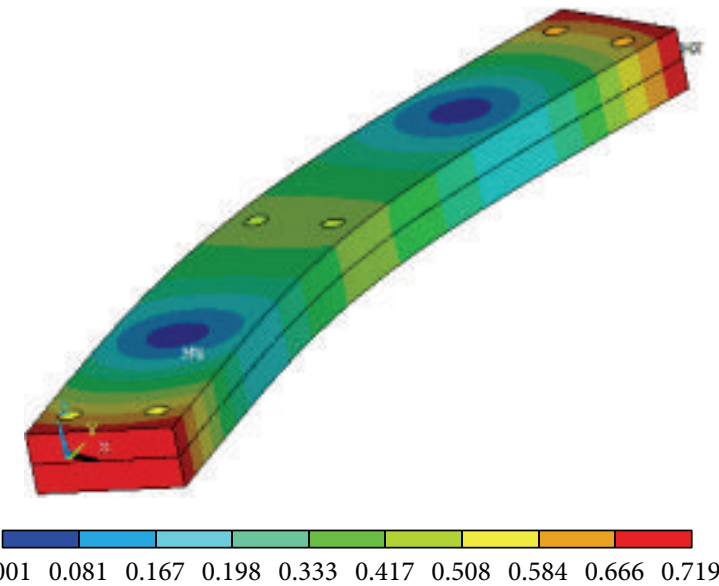

(d) 4th vibration mode

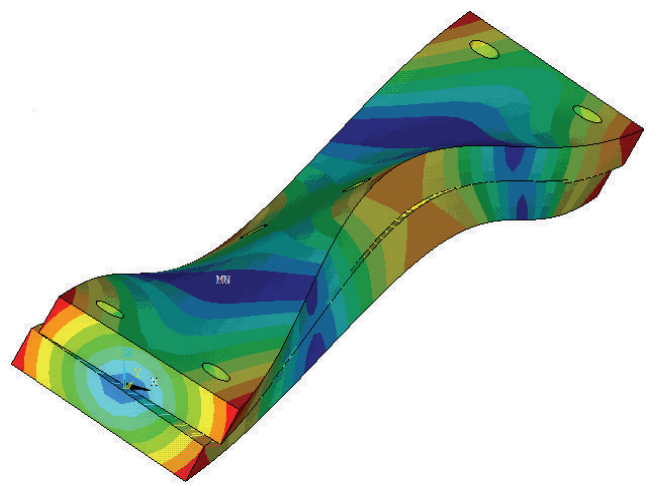

$\begin{array}{llllllllll}0.012 & 0.120 & 0.228 & 0.336 & 0.444 & 0.552 & 0.660 & 0.768 & 0.876 & 0.984\end{array}$

(f) 6th vibration mode

Figure 14: Model analysis results.

area, and the relationship is nonlinear, especially for normal damping coefficient. The most significant factors that affect joint surfaces' dynamics are normal stress, surface roughness, and topography. Normal stiffness increases significantly along with normal stress but normal damping coefficient decreases slightly when normal stress increases. The better the surface quality is, the higher the normal stiffness is; the more uniform the topography is, the higher the normal stiffness is; meanwhile the normal damping coefficient increases to a certain value and then decreases. 


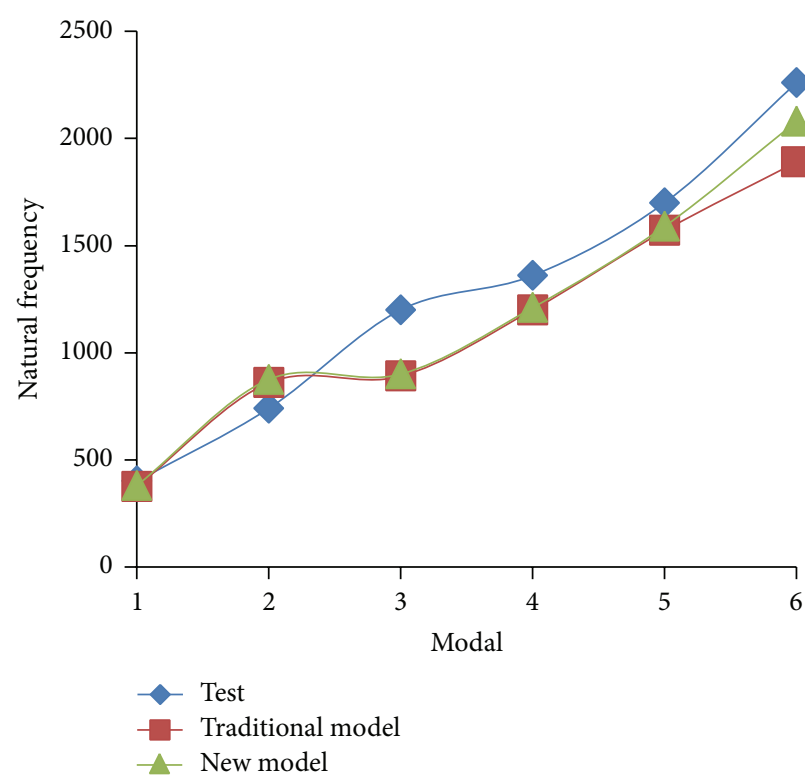

(a)

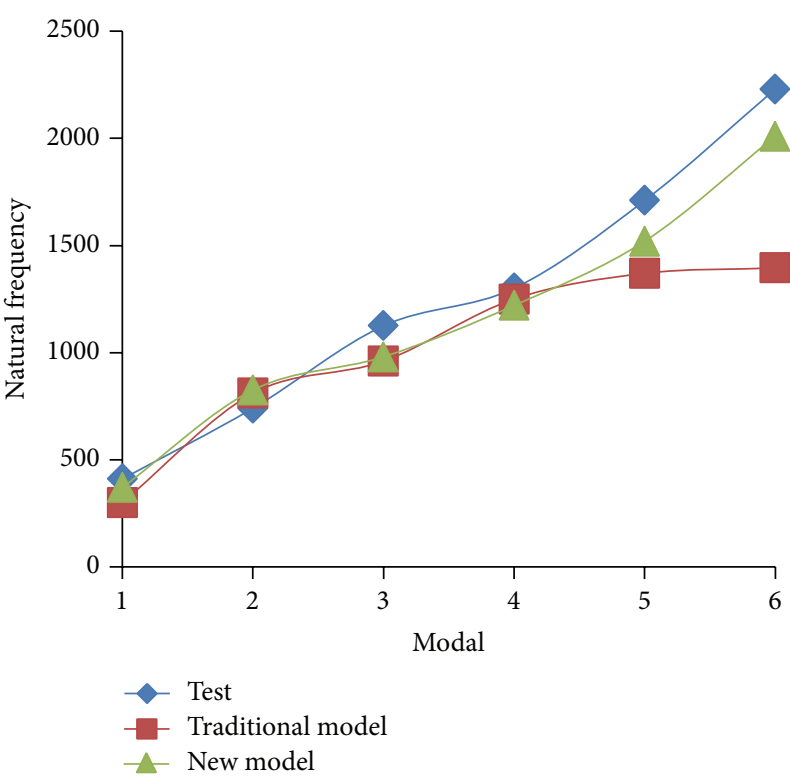

(b)

FIGURE 15: Simulation results comparison.

(3) Simulation and experiment results also show that stress distribution has some degree of influence on dynamic properties of joint surfaces, especially on high order modals when stress distribution becomes more uneven.

\section{Conflict of Interests}

The authors declared that they have no conflict of interests regarding this work.

\section{Acknowledgment}

This work was financially supported by the National Natural Science Foundation of China (Grant no. 51205087).

\section{References}

[1] Y. Ren and C. F. Beards, "Identification of 'effective' linear joints using coupling and joint identification techniques," Journal of Vibration and Acoustics, Transactions of the ASME, vol. 120, no. 2, pp. 331-338, 1998.

[2] R. A. Ibrahim and C. L. Pettit, "Uncertainties and dynamic problems of bolted joints and other fasteners," Journal of Sound and Vibration, vol. 279, no. 3-5, pp. 857-936, 2005.

[3] C. Andrew, J. A. Cockburn, and A. E. Waring, "Metal surfaces in contact under normal forces: some dynamic stiffness and damping characteristics," Proceedings of the Institution of Mechanical Engineers, vol. 182, pp. 92-100, 1967.

[4] H. Yumei, "An analytic method of joint condition damping parameters in dynamic design of mechanical system," in Proceedings of the 3rd International Conference on High Technology, pp. 511-515, Chiba, Japan, 1992.

[5] J. T. Oden and J. A. Martins, "Models and computational methods for dynamic friction phenomena," Computer Methods in Applied Mechanics and Engineering, vol. 52, no. 1-3, pp. 527634, 1985.

[6] R. E. Schofield and R. H. Thornley, "Mathematical expression of surface-finish characteristics," in Proceedings of the Conference on Properties and Mechanical Engineers, 1968.

[7] M. Zatarain and F. le Maître, "Behaviour of covering materials for guideways," CIRP Annals-Manufacturing Technology, vol. 38, no. 1, pp. 389-392, 1989.

[8] H. A. Sherif, "Investigation on effect of surface topography of $\mathrm{pad} / \mathrm{disc}$ assembly on squeal generation," Wear, vol. 257, no. 7-8, pp. 687-695, 2004.

[9] H. A. Sherif, "Parameters affecting contact stiffness of nominally flat surfaces," Wear, vol. 141, no. 1, pp. 113-121, 1991.

[10] V. N. Kirsanova, “The shear compliance of flat joints," Machines and Tooling, vol. 38, pp. 30-34, 1967.

[11] M. Masuko, Y. Ito, and C. Fujinoto, "Behaviour of horizontal stiffness and micro-sliding on the bolted joint under the normal pre-load," in Proceedings of the 12th International Machine Tool Design and Research Conference (MTDR '72), 1972.

[12] P. F. Rogers and G. Boothroyd, "Damping at metallic interfaces subjected to oscillating tangential loads," Journal of Engineering for Industry -Transactions of the ASME, vol. 97, no. 3, pp. 10871093, 1975.

[13] D. O. Smallwood, D. L. Gregory, and R. G. Coleman, Damping Investigations of a Simplified Frictional Shear Joint, Engineering Sciences Center Sandia National Laboratories, 2001.

[14] S. Bograd, P. Reuss, A. Schmidt, L. Gaul, and M. Mayer, "Modeling the dynamics of mechanical joints," Mechanical Systems and Signal Processing, vol. 25, no. 8, pp. 2801-2826, 2011.

[15] A. Majumdar and B. Bhushan, "Fractal model of elastic-plastic contact between rough surfaces," Journal of Tribology, vol. 113, no. 1, pp. 1-11, 1991.

[16] A. Majumdar and B. Bhushan, "Role of fractal geometry in roughness characterization and contact mechanics of surfaces," ASME Journal of Tribology, vol. 112, no. 2, pp. 205-216, 1990. 

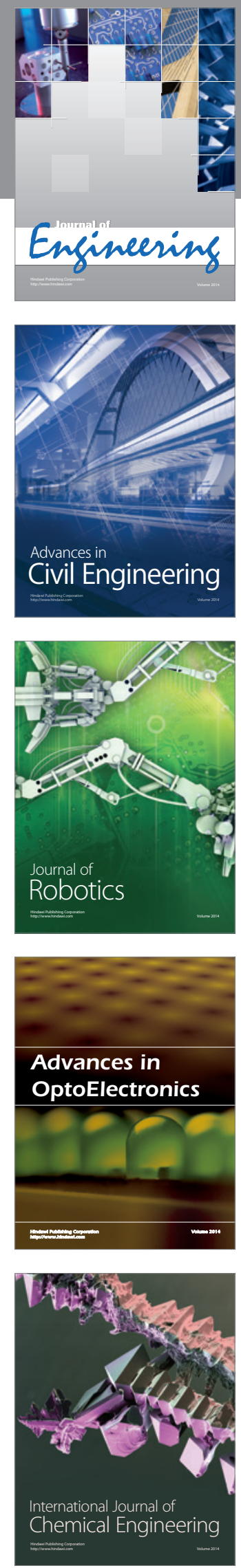

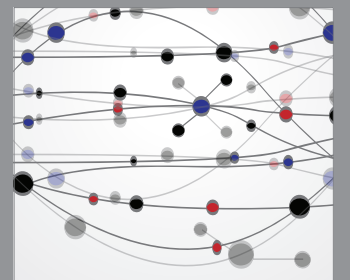

The Scientific World Journal
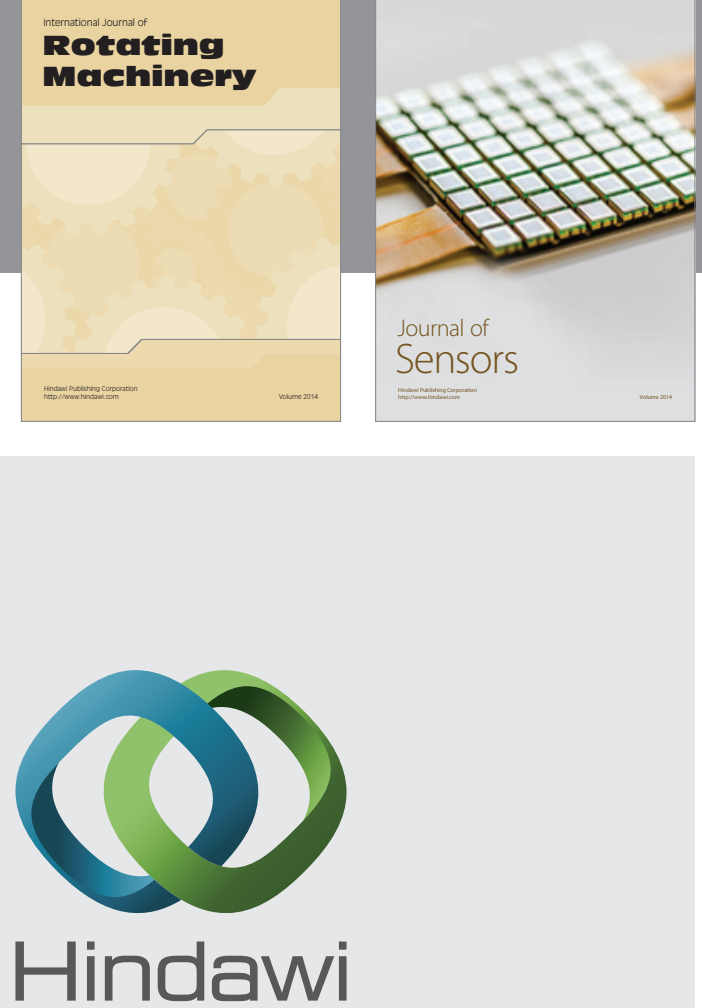

Submit your manuscripts at http://www.hindawi.com
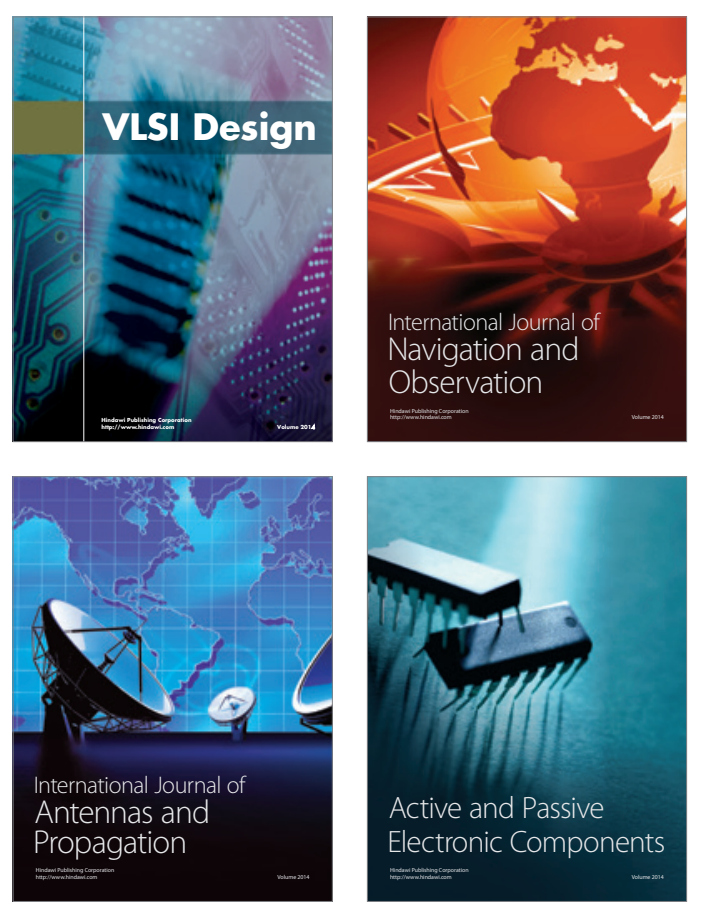
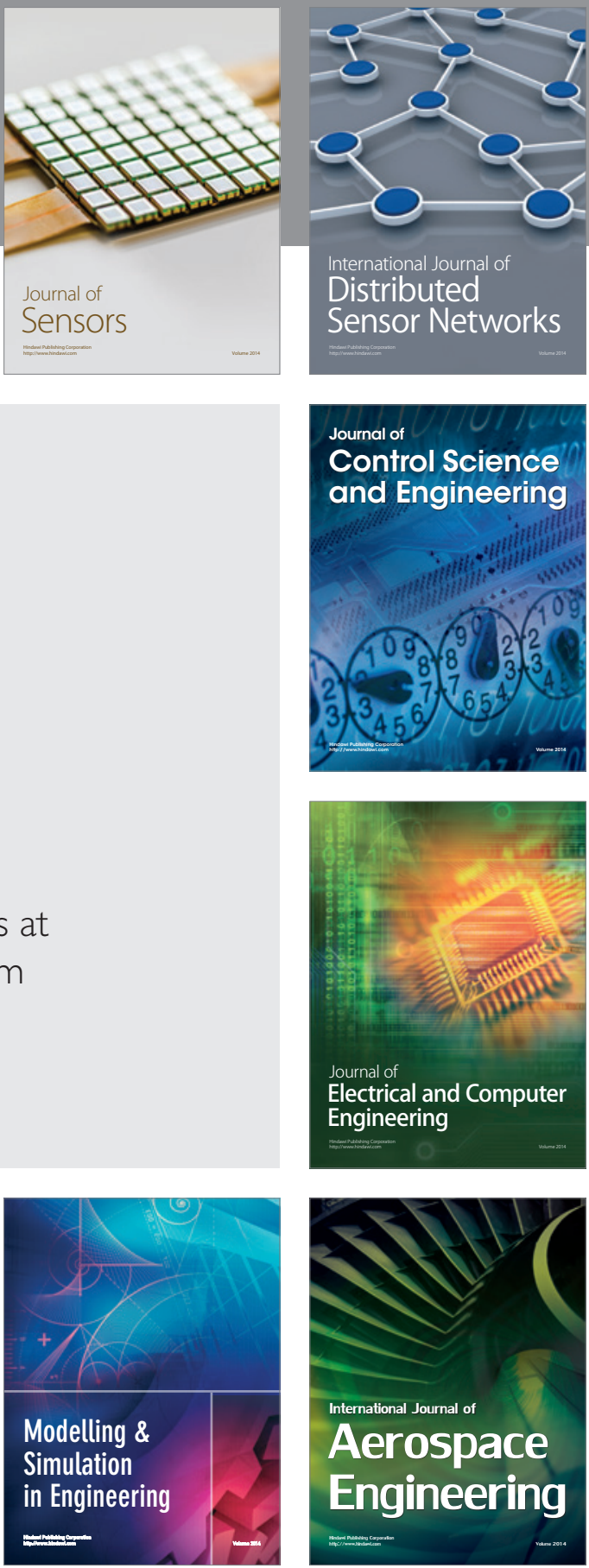

Journal of

Control Science

and Engineering
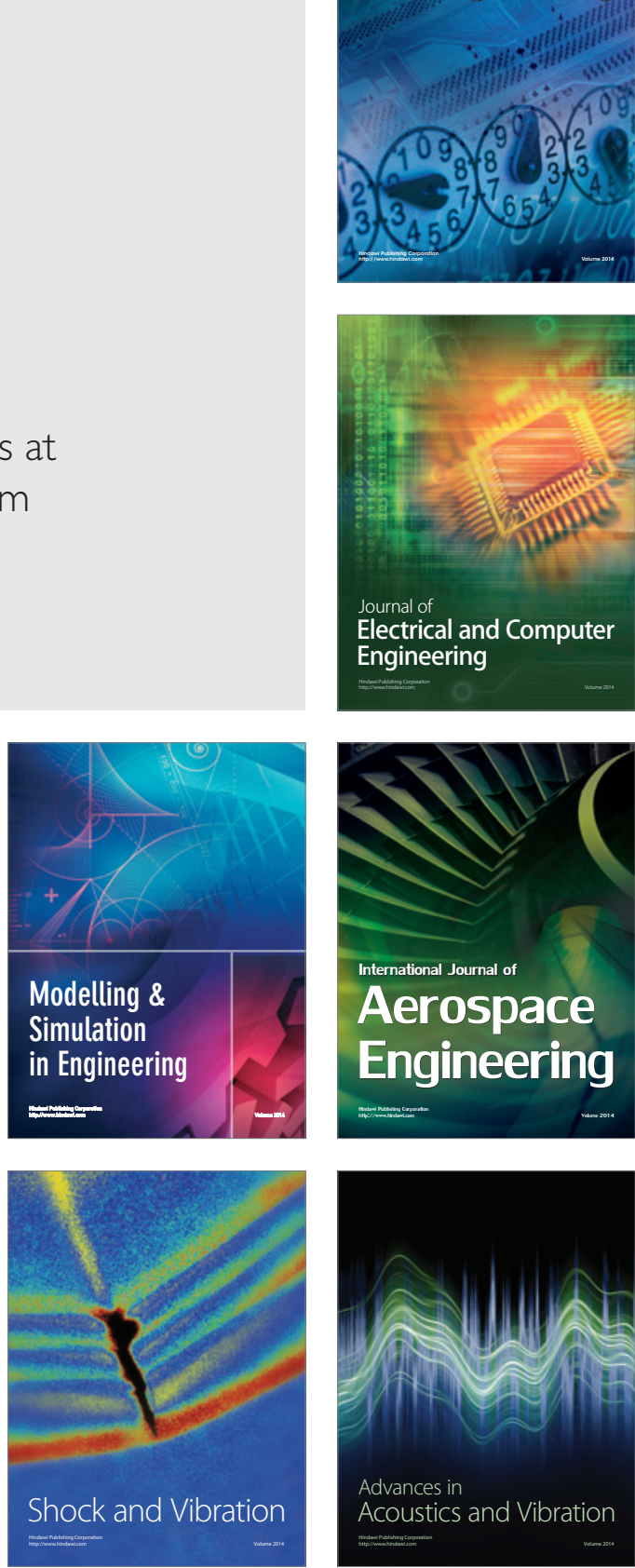